\title{
La sobrecualificación en el sector turístico: el caso español (1987-2011)
}

\author{
J. ROSa MARRERO RODRÍGUEZ \\ Departamento de Sociología \\ Instituto Universitario de Ciencias Políticas y Sociales \\ Universidad de La Laguna \\ jrmarrod@ull.es
}

Recibido: 28-02-2013

Aceptado: 07-02-2014

\begin{abstract}
RESUMEN
Este trabajo se sitúa en el ámbito de las relaciones entre formación y empleo en el sector turístico. Se parte de varias evidencias: las cualificaciones en el sector turístico se han generado históricamente en la empresa; en este sector es frecuente el bajo porcentaje de titulados universitarios. Por otro lado, en los últimos veinte años la universidad española ha crecido de forma espectacular.

El propósito de este texto es analizar la situación de los titulados universitarios en el sector turístico. El incremento de los egresados universitarios en el mercado laboral turístico es evidente, pero queda por saber qué espacios laborales concretos ocupan en dicho sector. Todo ello se hará utilizando los datos de la Encuesta de Población Activa. Se intenta responder a la cuestión de si aspectos institucionales como el incremento de los egresados universitarios afecta a la estructura ocupacional del sector, y por tanto a su conformación como mercado de trabajo secundario.
\end{abstract}

Palabras clave: formación universitaria, empleo, sector turístico, mercado de trabajo.

\section{The overqualification in the touristic activity: the Spanish case (1987-2011)}

\begin{abstract}
This research is about the relationships between education and employment in the touristic sector. It is known that the qualifications in the tourism sector have historically generated in the company, and the low percentage of university graduates is usual. But in the last twenty years it is also important that the Spanish university has grown dramatically. The purpose of this paper is to analyze the situation of graduates in tourism. The increase of university graduates in the tourism labour market is evident, but it remains to be known the specific positions they held. This will be done using data from the Spanish Labour Force Survey. This research aims to find out whether the institutional aspects affect the occupational structure of the sector and therefore its conformation as a secondary labour market.
\end{abstract}

Keywords: university education, employment, tourism, labour market.

\section{REFERENCIA NORMALIZADA}

Marrero Rodríguez, J. R. (2013). “La sobrecualificación en el sector turístico: el caso español (1987-2011)”. Cuadernos de Relaciones Laborales, Vol. 33, núm. 1, p. 149-168. 
SUMARIO: Introducción. 1. Antecedentes. 2. Cuestiones previas de carácter metodológico. 3. La incorporación de egresados universitarios al sector turístico público. 4. La ocupación de los universitarios en el sector turístico. 5. Reflexiones finales. 6. Bibliografía.

\section{Introducción}

Este trabajo se sitúa en el ámbito de las relaciones entre formación y empleo en el sector turístico. A lo largo de las últimas décadas, muchas de las cualificaciones del sector turístico se han desarrollado en el mismo puesto de trabajo y/o mediante la rotación entre empresas, dando lugar a lo que Pries (1988) denominó para el sistema productivo español como 'estrechez del ámbito empresarial’; por otro lado, es común en el sector el bajo porcentaje de titulados universitarios, así como que esto tiene que ver con las características económico-tecnológicas del mismo.

También es cierto que, en los últimos veinte años, la universidad española ha crecido de forma espectacular (Cervera-Taulet y Ruiz-Molina, 2008; Mallorquí Ruscalleda, 2007). Y desde los años noventa ha integrado los títulos de turismo, lo que ha favorecido el crecimiento del número de centros que ofertan la diplomatura de turismo y, por consiguiente, también aumento importante de los egresados en dicha titulación. Más recientemente, en el marco del espacio superior europeo, las diplomaturas de turismo han pasado a ser grados de cuatro años (Ceballos Hernández y otros, 2010; García Manjón y Pérez López, 2008). El propósito de este texto es analizar la situación de los titulados universitarios en el sector turístico.

La mejora del sistema educativo español ha contribuido a que el porcentaje de personas con educación formal que se incorpora al mercado laboral sea cada vez mayor. Ahora bien, las características del sector turístico y la propia dinámica interna del sistema educativo, sobre todo el universitario, dificultan la creación de perfiles profesionales específicos (Cervera-Taulet y Ruiz-Molina, 2008). Ello se traduce en un incremento de los egresados universitarios en el mercado laboral turístico. Pero queda por saber cómo se produce la incorporación de titulados universitarios en sectores que, tradicionalmente y por sus características internas, presentan bajos porcentajes de titulados. El incremento de egresados universitarios que se incorporan al mercado laboral también afecta al sector turístico, y el objetivo de este trabajo es contribuir a conocer qué puestos laborales ocupan. Para ello se tomará como año de partida 1987, y se realizará la comparación con 2009 y con 2011 (según para qué variables). Los datos proceden de la Encuesta de Población Activa. Si el incremento de universitarios contribuye (o no) a la productividad o si se adecúa a las demandas de las empresas forma parte de otro trabajo.

Parece razonable, en cualquier caso, proceder a desmitificar la importancia de la formación en el sector turístico. De ignorarla, incluso cuando su importancia económica ya era evidente en España, se ha pasado a defenderla con pasión más recientemente; mientras en las empresas se siguen observando situaciones de lo más variadas: desde cadenas hoteleras, restaurantes o agencias de viajes comprometidas con la formación a aquellas cuyas condiciones laborales y de contratación dificultan la formación. Por lo que ¿para quién es clave la formación en el sector? ¿para las 
empresas cuyo objetivo es mantener el producto de sol y playas que tanto rédito ha dado, o bien para aquellas con interés en los nuevos tipos de turistas? ¿se beneficia la administración, en un contexto de creación tan raquítica de empleo? Quizá la importancia dada a la formación por los expertos académicos y por la opinión pública no sea paralela a la que le concede el conjunto del sistema productivo, tanto cualitativa como cuantitativamente. Por ello, en este trabajo el interés por analizar el paradero de los universitarios tiene que ver con un compromiso que pretende ser realista con las “posibles y adecuadas” relaciones entre formación y empleo.

\section{Antecedentes}

Antes de continuar, conviene resumir brevemente el conjunto principal de afirmaciones que trabajos previos han subrayado sobre el empleo en el sector turístico. En lo que se refiere a las características de los individuos, se observa una mayor presencia proporcional de mujeres, jóvenes y de extranjeros; estamos en un sector complejo en la conformación de los puestos, porque son diversas las competencias a desempeñar: las competencias técnicas se entremezclan con las comportamentales, estéticas y actitudinales. Una de las consecuencias de esta convivencia es que hay competencias muy importantes que están poco reconocidas y formalizadas, lo que a su vez afecta a los salarios.

En el sector, los espacios laborales considerados de baja cualificación son frecuentes; ello favorece la presencia de personas con bajos niveles educativos. Por otro lado, debido al carácter inestable de la demanda, hay mayor número de contratos a tiempo parcial, temporales y turnos partidos. Todo ello afecta negativamente a las condiciones de trabajo (Álvarez Aledo, 1996; Airey y Frontistis, 1997; Baum, 1995, 2007; García Pozo y otros, 2011; Ladkin y Riley, 1996; Lam, 2002; Lee y Kang, 1998; Lennon y Wood, 1989; Lever, 1987; Liu y Wall, 2005; Lucas, 2004; Pizam, 1999; Riley y Szivas, 2003; Riley, Ladkin y Szivas, 2002; Rodríguez Rodríguez y Arroyo Varela, 2000; Servicio Público de Empleo Estatal, 2011; Sheldon, 1989; van den Berghe, 1992). De aquí se deriva la imagen de un sector con bajas cualificaciones.

También tenemos en cuenta en este trabajo conclusiones más recientes de otros trabajos como los de Lillo-Bañuls y Casado Díaz (2011: 764), quienes concluyen que

Los rendimientos que los ocupados obtienen a cambio de su inversión en formación reglada es mucho menor en el caso de las actividades turísticas que en la economía considerada en su conjunto, y que la diferencia es especialmente notable en el subsector de hostelería y restauración.

Las conclusiones del presente trabajo se podrían considerar como marco explicativo complementario del asunto relativo al menor rendimiento del capital humano del que hablan estos autores. También se aborda en el trabajo de Lillo- 
Bañuls y Casado Díaz la sobreeducación de los universitarios que trabajan en el sector objeto de estudio.

Por otro lado, se ha tenido en cuenta el enfoque de carácter cualitativo de Brotons Martínez (2012), que concluye, entre otros, con la importancia de la experiencia y de los idiomas que los empresarios turísticos atribuyen en el momento de la contratación.

Otra línea de trabajo y reflexión que se ha tenido en cuenta matiza parcialmente lo planteado anteriormente. Según Ladkin (2011), hay evidencia empírica que sugiere que muchas de las personas que trabajan en el sector tienen altos niveles educativos, pese a que muchas de las ocupaciones en el sector turístico requieren bajas cualificaciones. Pero entendido desde una perspectiva culturalista, que considera cómo en la nueva economía las relaciones entre las personas, el capital humano y sus dimensiones están cambiando. Por la naturaleza cuantitativa de los datos que se van a manejar sólo se retomarán estas reflexiones en las conclusiones.

Antes de continuar, conviene además hacer referencia al caso español. Pese a que en la comparativa internacional se han observado paralelismos en cuanto a las condiciones laborales de este sector, España presenta algunas particularidades, producto de sus recientes condiciones socio-históricas. Hasta la crisis que da comienzo en 2008, el sector turístico y el de la construcción han ido de la mano en muchas comunidades autónomas. Esta circunstancia tiene que ver con la importancia de la fase constructiva en las zonas turísticas, con el desarrollo de productos turísticos para los que es central la edificación y remodelación de los espacios (segunda residencia, campos de golf), y con circunstancias propias de las necesidades y demandas de vivienda en España. Todo ello hizo espectacular el crecimiento del parque de viviendas antes del comienzo de la crisis -y está íntimamente vinculado a la misma- (Mazón Martínez y otros 2009, Serrano Martínez, 2011).

Resulta relevante esta reflexión para los objetivos de este trabajo, puesto que la presencia combinada de oportunidades laborales en la construcción y el turismo en muchas comunidades autónomas puede haber actuado como elemento disuasorio para muchas personas en la búsqueda de mejoras educativas, y para empresarios en el emprendimiento de actividades económicas de mayor valor añadido; también puede haber afectado a las prácticas de selección y contratación en las empresas. Porque la acción combinada de construcción y turismo da lugar a un mayor número de ofertas de empleo de baja y media cualificación.

El planteamiento realizado aquí no aborda los salarios, sino los puestos, y por tanto la estructura ocupacional. Partiendo de la idea de que las posiciones de los individuos en las empresas son fundamentales para comprender sus condiciones laborales. La cuestión no es si debe haber más o menos universitarios en el sector turístico, pues esto lo ‘determinan’ condiciones económico-tecnológicas específicas y los juegos de relaciones empresa-sindicatos-empleados en el marco establecido por dichas condiciones (Villa, 1990); lo que implica que las condiciones tecnológicas y organizativas demandarán mayor o menor número de titulados. La cuestión que interesa responder es dónde trabajan los universitarios, en qué espacios 
ocupacionales concretos se insertan: son directivos, ocupan puestos de profesionales o técnicos, son recepcionistas, son cocineros, etc.

Por todo lo anterior, se plantean dos hipótesis en este trabajo:

1. La mejora del sistema educativo español ha repercutido en un incremento de titulados universitarios en el sector turístico, aunque no con la intensidad que se observa para el conjunto de la población ocupada. Tiene sentido este supuesto porque las características estructurales del sector dificultan que los universitarios sean tan importantes como en la media del conjunto ocupacional. Por ello, el crecimiento de titulados superiores se producirá de tal forma que mantendrá las distancias entre el sector turístico y la media de la población ocupada.

2. Este incremento de los ocupados universitarios favorece a los grupos directivos y profesionales del sector, pues son los puestos de los que cabe esperar mayor demanda de dichas titulaciones. Esta hipótesis se formula a partir de planteamientos teórico-empíricos. Por un lado, muchas de las ocupaciones que conforman el grupo de directivos y de profesionales exigen un título universitario para el ejercicio profesional (véase la clasificación nacional de ocupaciones). Por otro, la literatura dedicada a la sobreeducación subraya que ésta disminuye con la movilidad, siendo parte de la misma la ascendente en las empresas (Blázquez Cuesta y Mora, 2010; Marchante Mera y otros, 2004; Sala, 2011). Podemos considerar que la sobreeducación tiende a disminuir al buscar los empleados opciones laborales más adecuadas a los títulos obtenidos y los empresarios personas que puedan aportar mayor formación al puesto. Por ello, las empresas aprovechan el incremento de titulados, y es más probable que los puestos directivos y profesionales cuenten con un mayor número de universitarios.

Las ideas que fundamentan estas hipótesis están vinculadas a la literatura de los mercados secundarios de trabajo (Álvarez Aledo, 1996) y a los enfoques institucionalistas que inciden en la importancia de las dimensiones tecnoeconómicas e institucionales para comprender los mercados de trabajo (Villa, 1990). Los sectores de actividad presentan diferencias por los fundamentos económicos, tecnológicos e institucionales propios de los mismos. De lo que se trata es de aplicar algunos de estos planteamientos al sector turístico, especialmente las consecuencias inesperadas del incremento de egresados universitarios en un sector cuya cualificación se ha llevado a cabo tradicionalmente en el mismo puesto de trabajo.

\section{Cuestiones previas de carácter metodológico}

La Encuesta de Población Activa se publicó por primera vez en 1964. En 1987 se modificó el cuestionario de la encuesta para adaptarse a las recomendaciones internacionales de entonces, dando lugar a la denominada 'serie homogénea' de la EPA que ofrece actualmente el Instituto Nacional de Estadística (www.ine.es). 
Desde entonces ha sufrido cambios metodológicos que afectan a distintos contenidos, como es el concepto de paro.

En cuanto a las ocupaciones, de 1979 hasta 1994 se utilizó la clasificación nacional de ocupaciones denominada CNO79; desde 1994 hasta 2010, la CNO94. A partir del primer trimestre de 2011 se introduce en la EPA la nueva clasificación de ocupaciones CNO-2011. Esta nueva clasificación modifica la composición de los grandes grupos de ocupaciones de la CNO94, al igual que la consideración de cualificación o profesionalidad presente en la anterior clasificación. Sin embargo, las diferencias entre CNO79 y CNO94 son menores y no afectan al ejercicio que se va a desarrollar a continuación.

Para proceder al análisis de los cambios en el sector turístico mencionados, se selecciona 1987 como situación más antigua (segundo trimestre); y se toma 2009 como año más reciente. Sin embargo, para el desarrollo del primer apartado que no tiene en cuenta las ocupaciones sino el nivel educativo de los individuos ocupados en el sector turístico, se llega hasta 2011 puesto que los ítems de las variables estudiadas son comparables.

Por último, conviene señalar que se ha trabajado con una variable denominada por el INE 'condición socioeconómica' (CSE), que "es una variable con la que se pretende clasificar a los encuestados en grupos de población que sean razonablemente homogéneos en cuanto a las características sociales, económicas y culturales. La CSE se obtiene combinando las variables ocupación, situación profesional y en algunos casos ramas de actividad y estudios” (INE). Por ello, aunque la variable utilizada en este trabajo es la condición socioeconómica, ésta requiere de la ocupación para su elaboración, por lo que resultaba importante que se mantuviera la homogeneidad en la clasificación de la misma. Ésta es la razón por la que, para la segunda hipótesis, nos detenemos en 2009.

\section{La incorporación de egresados universitarios al sector turístico español}

El sistema educativo español ha mejorado profundamente en los últimos treinta años. Y aunque es cierto que los titulados universitarios que se incorporan al sector turístico proceden de diversas especialidades, conviene tener presente la historia reciente de los estudios turísticos en España (véase el Libro Blanco del Grado de Turismo, elaborado por la ANECA).

A partir de los datos de la Encuesta de Población Activa, se observa una mejora notable de los niveles educativos de la población ocupada en España, pero mucho mayor en el sector de hostelería. Mientras que el sector de alojamiento y restauración partía en 1987 de un nivel muy bajo de titulados universitarios $(1,9 \%)$, el conjunto de la población ocupada española ya presentaba un 10,6\%. El conjunto de la población ocupada terminará 2011 con un 27\% de universitarios y el sector objeto de estudio con un 10,3\%. Las tendencias son de crecimiento paulatino a partir de los años noventa (gráfico 1). 
Ahora bien, aunque la presencia de titulados universitarios sigue siendo baja en el sector de alojamiento y restauración, es espectacular el crecimiento que ha tenido (de más del 1.200\%), si lo comparamos con lo ocurrido en el total de ocupados en el sector, con los universitarios en el conjunto de la población ocupada, y con la propia evolución del total de ocupados. Aunque ha aumentado la importancia relativa del sector de alojamiento y restauración en el total de ocupados españoles (que pasa del 5,1\% al 8,1\%); y también lo ha hecho la presencia relativa de titulados universitarios en alojamiento y restauración en el subgrupo de ocupados universitarios, pasando del 0,9\% en 1987 al 3,1\% en 2011; lo cierto es que es impresionante el crecimiento de universitarios en el sector objeto de estudio (véase la tabla 1).

Por otro lado, es evidente que el sector turístico se ha alimentado no sólo de los egresados de las diplomaturas de turismo, sino de todas las restantes titulaciones del sistema educativo español. Como conclusiones provisionales, tenemos el importante crecimiento de universitarios en el sector turístico, con unas tasas de crecimiento impresionantes. Sin embargo, se mantienen las distancias con respecto a lo que ocurre en el conjunto de la población ocupada, e incluso aumentan las mismas. Esto se da en un contexto en el que el sector turístico alcanza una mayor presencia en el entramado productivo español.

\section{Gráfico 1. Proporción de titulados universitarios en alojamiento- restauración y en el total de la población ocupada, España 1987-2011}

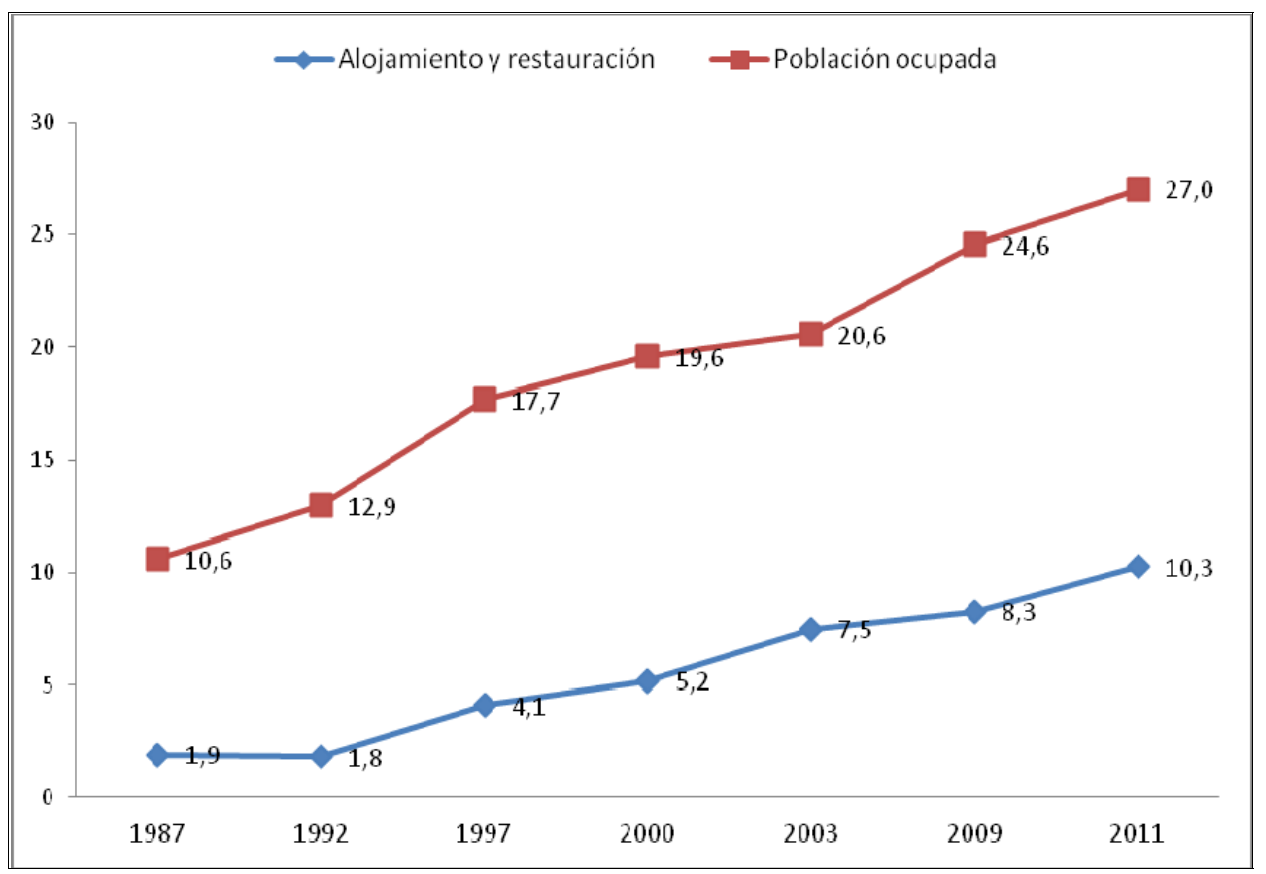

Fuente: Elaboración propia con microdatos de EPA 
Tabla 1. Población ocupada total y en subsector alojamiento-restauración, y universitarios en cada grupo, España 1987 y 2011

\begin{tabular}{|l|r|r|r|}
\hline & $\mathbf{1 9 8 7}$ & $\mathbf{2 0 1 1}$ & $\begin{array}{c}\text { Crecimiento } \\
\text { relativo }\end{array}$ \\
\hline Total ocupados & 11.254 .876 & 18.156 .255 & $61,3 \%$ \\
\hline $\begin{array}{l}\text { Total ocupados con titulación universita- } \\
\text { ria }\end{array}$ & 1.191 .915 & 4.903 .737 & $311,4 \%$ \\
\hline $\begin{array}{l}\text { Total ocupados en subsector de aloja- } \\
\text { miento y restauración }\end{array}$ & 581.447 & 1.485 .016 & $155,4 \%$ \\
\hline $\begin{array}{l}\text { Total ocupados con titulación universita- } \\
\text { ria en subsector alojamiento y restaura- } \\
\text { ción }\end{array}$ & 11.119 & 152.770 & $1.273,9 \%$ \\
\hline
\end{tabular}

Fuente: Elaboración propia con microdatos de EPA

La primera hipótesis se confirma, pero sólo si tenemos en cuenta el espectacular crecimiento de los universitarios en el sector objeto de estudio. El contexto institucional influye en las características de los sectores productivos, modificando sus constantes estructurales. Y ello es así porque la existencia de un sistema educativo, que proporciona un número creciente de personas tituladas, altera la situación de sectores laborales caracterizados históricamente por su raquítica presencia.

Sin embargo, queda por analizar a qué se debe este impresionante incremento. Dado que pueden ser diversas las razones: el sector demandaba y no podía cubrir previamente esas titulaciones; o bien la mejora del nivel educativo de los activos es aprovechada por los empleadores para cubrir puestos de tipo intermedio que también podría resolverse mediante otros títulos, o mediante la tradicional formación en el puesto. Incluso pueden darse situaciones intermedias. Como que el incremento de egresados es utilizado por las empresas para proceder a cambios organizativos internos. O que una parte de estos universitarios se encuentra en una situación transitoria, camino de una mejor opción laboral.

\section{La ocupación de los universitarios en el sector turístico}

Queda por responder a la segunda de las hipótesis planteadas, la relativa al destino de los titulados universitarios en las empresas. Para ello vamos a trabajar con la categoría 'condición socio-económica' que utiliza la EPA, y con el período 1987-2009. Como se puede observar en el gráfico 2, la estructura ocupacional del sector alojativo (ahora no se incluye restauración) permanece relativamente estable a lo largo de más de veinte años, siendo mínimas las variaciones. Dicha estructura ocupacional está escasamente profesionalizada, con porcentajes de directivos y empleadores reducidos, y también pequeños grupos de operarios cualificados y no cualificados. La estructura ocupacional concentra a la mayor parte de los individuos en la parte central, con personal administrativo y de servicios sumando algo más de 
las tres cuartas partes de los ocupados. En 1987 el porcentaje de puestos profesionales se situaba en el $1 \%$ mientras que en el conjunto de la población ocupada superaba el $8 \%$.

El caso español, por otro lado, es relativamente parecido al de otros muchos países europeos, en lo que a la estabilidad estructural de los componentes ocupacionales se refiere. Véase al respecto el documento de Eurofound (2009).

Ahora bien, pese a la estabilidad de la estructura ocupacional, se trata de una población que ha crecido un 149,3\%, destacando tres colectivos; los profesionales crecen un 960,4\%, los directivos un 283,3\% y los operarios cualificados un 168,2\%. En el resto de los grupos, el crecimiento no es tan importante (véase la tabla 2).

En definitiva, en estas últimas décadas las empresas de alojamiento turístico han ensanchado su parte central, manteniendo el peso del colectivo de trabajadores de servicios cualificados, pero con una tendencia a una mayor cualificación, dado que los administrativos y profesionales mejoran su presencia. Al tiempo que los grupos localizados en los extremos, empresarios y operarios no cualificados observan una disminución.

¿Qué ocurre con los titulados universitarios? Este colectivo se concentraba en 1987 en el grupo de empleadores (14\%), directivos (26,4\%), profesionales $(14,5 \%)$ y administrativos (19,6\%). Sumando estos cuatro colectivos casi las tres cuartas partes. En 2009, estos cuatro grupos sólo suman el 30,2\%. Y es que en 2009, el $62,4 \%$ de los universitarios se concentra en el grupo personal de servicios. Se preveía que el increíble crecimiento de los titulados en el sector beneficiaría a directivos, empleadores y profesionales. No ha sido así. La estructura ocupacional de los universitarios es también de 'clase media', está en la mitad de la jerarquía profesional. Esto se puede observar en el gráfico de proporción de universitarios en cada grupo de condición socio-económica (gráfico 3).

La imagen que ofrece dicho gráfico es desconcertante. Teniendo en cuenta el gráfico 3 y la tabla 2, mientras que los directivos y profesionales presentaban una alta proporción de universitarios en 1987, en 2009 ha disminuido su presencia relativa. Por otro lado, empleadores y autónomos mejoran, llegando estos cuatro grupos casi a una cuarta parte de universitarios. Y es el colectivo de administrativos al que mejor le va, pasando de un $8 \%$ a un $32 \%$ de universitarios.

La imagen resultante parece problematizar la resolución de la segunda hipótesis. Por un lado, el sector se profesionaliza, al beneficiar el crecimiento del empleo al grupo de profesionales, y algo menos directivos y administrativos. Por otro lado, el crecimiento de los universitarios es mayor que el del resto de grupos educacionales. Y finalmente, estas dos situaciones contrastan con el hecho de que sean los grupos administrativos y personal de servicios donde más crezcan los universitarios. Éstos se ubican más en la zona intermedia de la estructura ocupacional que en la superior.

Todo ello tiene como consecuencia el que los universitarios estuvieran a finales de los ochenta más repartidos entre directivos, profesionales, empleadores y administrativos de lo que es la situación posterior. En 2009 el 80\% se concentra en los grupos de administrativos y personal de servicios. La tesis de la sobreeducación parece encontrar acomodo con estos datos. Desde luego, el incremento de titulados 
en el sector no ha servido para mejorar la formación universitaria de directivos y profesionales. Beneficia a los administrativos y trabajadores cualificados.

Si se profundiza en los datos, se obtiene algo más de información sobre estos procesos de inserción laboral de los activos universitarios. Si comparamos la situación de las ocupaciones de los universitarios españoles en el conjunto de la población ocupada, las diferencias son notables. En el segundo trimestre de 1987, el $68,3 \%$ de todos los ocupados universitarios eran considerados profesionales, mientras que era el caso de sólo el 14,5\% en el sector alojativo. En 2009 había empeorado, pues el $63 \%$ de los universitarios en el total de ocupados eran profesionales, mientras que es el caso de sólo el 3\% en el sector objeto de estudio. La coherencia entre títulos y ocupaciones parece mucho menor en el sector turístico. En el conjunto de la población ocupada, los universitarios están mucho más vinculados al grupo de profesionales y técnicos de lo que se observa en el sector alojativo.

Si se atiende a la dimensión de género, en 1987 sólo el 18,4\% de los universitarios eran mujeres, mientras que en el sector ya alcanzaban el $44,3 \%$. Es radical el cambio en 2009, pues eran mujeres el $47,1 \%$ de los universitarios en ese sector (y $60 \%$ en todo alojamiento). Es por ello, que se considera, para finalizar este apartado, que quizá la dimensión de género pueda aportar algo de luz a estos procesos laborales.

Hasta qué punto esta concentración de universitarios en los grupos de administrativos y personal de servicios puede tener que ver con la dimensión de género, y en particular con el denominado 'techo de cristal', la dificultad de las mujeres de acceder a puestos profesionales y directivos en las empresas por razones de discriminación de género (Sarrió et al, 2002; Wirth, 2002; Witz, 1992). 
Gráfico 2. Distribución de los ocupados en el sector alojativo según condición socioeconómica, España 1987 y 2009

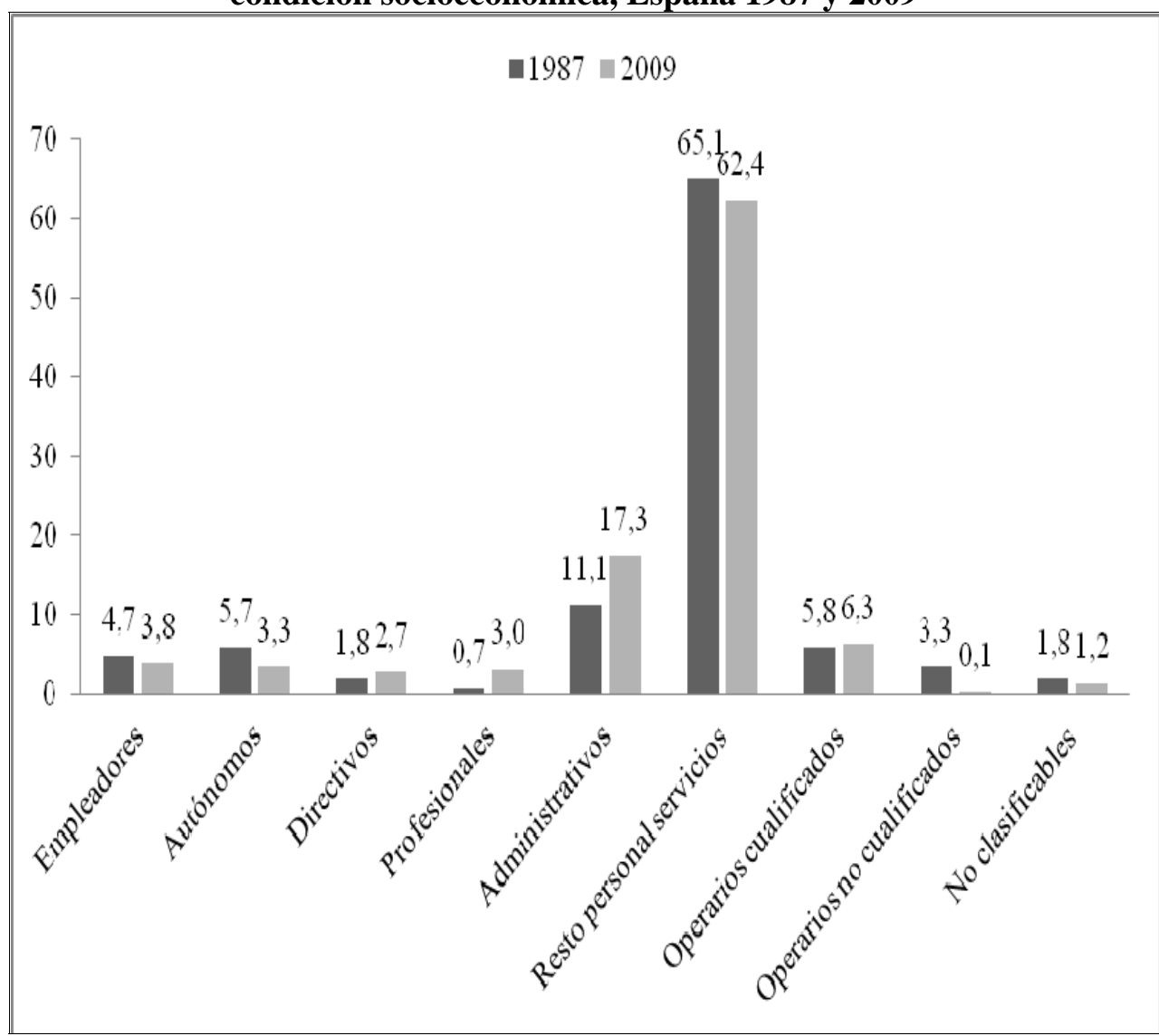

Fuente: Elaboración propia con microdatos de EPA 
Gráfico 3. Distribución de los ocupados con titulación universitaria en el sector alojativo según condición socioeconómica, España 1987 y 2009

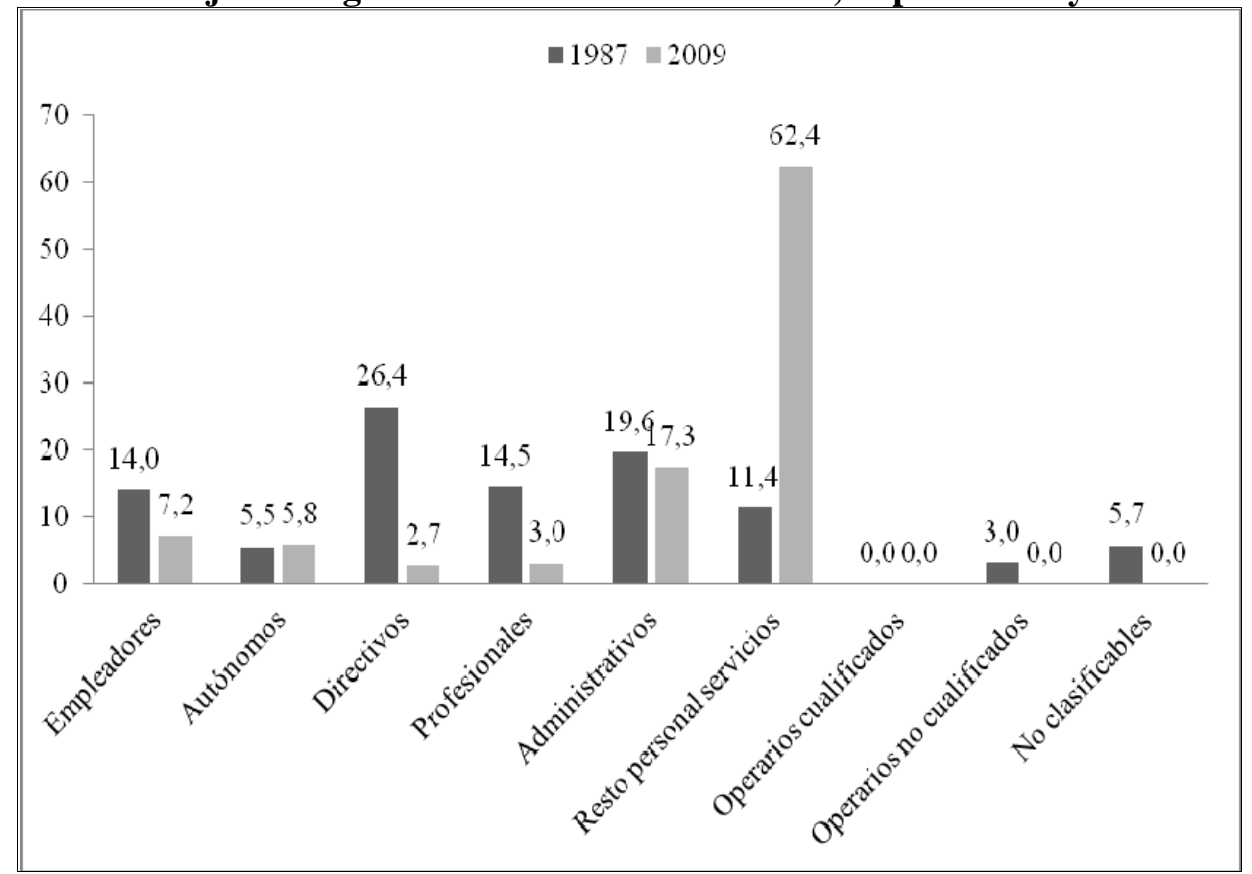

Fuente: Elaboración propia con microdatos de EPA 
Tabla 2. Población ocupada total y universitaria en el subsector alojativo en 1987 y 2009, España

\begin{tabular}{|c|c|c|c|c|c|c|}
\hline & \multicolumn{2}{|c|}{$\begin{array}{c}\text { Universitarios } \\
\text { en subsector } \\
\text { alojativo }\end{array}$} & \multirow[t]{2}{*}{$\begin{array}{l}\text { Creci- } \\
\text { miento } \\
\text { relativo } \\
\end{array}$} & \multicolumn{2}{|c|}{$\begin{array}{c}\text { Total ocupados en } \\
\text { subsector alojativo }\end{array}$} & \multirow[t]{2}{*}{$\begin{array}{l}\text { Crecimien- } \\
\text { to relativo }\end{array}$} \\
\hline & 1987 & 2009 & & 1987 & 2009 & \\
\hline $\begin{array}{l}\text { Empleado- } \\
\text { res }\end{array}$ & 868 & 3.046 & $250 \%$ & 6.128 & 12.134 & $98 \%$ \\
\hline Autónomos & 340 & 2.467 & $625,5 \%$ & 7.391 & 10.518 & $43,3 \%$ \\
\hline Directivos & 1.639 & 5.145 & $213,9 \%$ & 2.271 & 8.706 & $283,3 \%$ \\
\hline $\begin{array}{l}\text { Profesiona- } \\
\text { les }\end{array}$ & 902 & 4.731 & $424,5 \%$ & 902 & 9.565 & $960,4 \%$ \\
\hline $\begin{array}{l}\text { Administra- } \\
\text { tivos }\end{array}$ & 1.215 & 18.146 & $1.393,5 \%$ & 14.302 & 55.792 & $290 \%$ \\
\hline $\begin{array}{l}\text { Resto } \\
\text { personal } \\
\text { servicios }\end{array}$ & 706 & 8.925 & $1.164,1 \%$ & 83.936 & 200.589 & $138,9 \%$ \\
\hline $\begin{array}{l}\text { Operarios } \\
\text { cualificados }\end{array}$ & 0 & 0 & $0 \%$ & 7.495 & 20.106 & $168,2 \%$ \\
\hline $\begin{array}{l}\text { Operarios } \\
\text { no cualifi- } \\
\text { cados }\end{array}$ & 189 & 0 & $-100 \%$ & 4.234 & 294 & $-93 \%$ \\
\hline $\begin{array}{l}\text { No clasifi- } \\
\text { cables }\end{array}$ & 354 & 0 & $-100 \%$ & 2.336 & 3.900 & $66,9 \%$ \\
\hline Total & 6.213 & 42.460 & $583,4 \%$ & 128.995 & 321.604 & $149,3 \%$ \\
\hline
\end{tabular}

Fuente: Elaboración propia con microdatos de EPA 
Gráfico 4. Proporción de universitarios en cada uno de los grupos de condición socio-económica, España 1987 y 2009

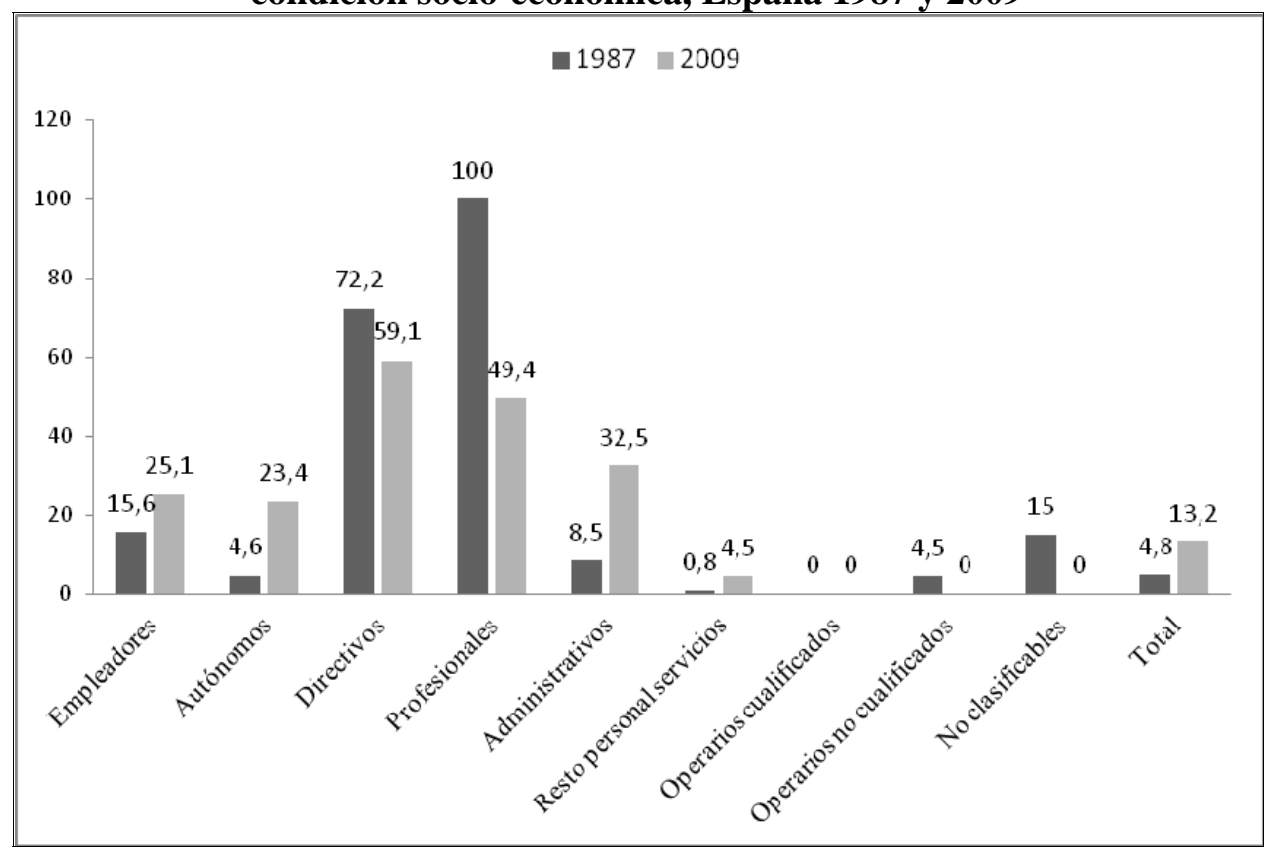

Fuente: Elaboración propia con microdatos de EPA

En el grupo de ocupados universitarios en el sector de alojamiento, las mujeres tienen una importante presencia en casi todos los grupos, excepto en el de empleadores. Más bien, parece producirse una situación anómala, en el contexto de la abundante bibliografía sobre discriminación laboral por razón de género, que aquí no tenemos tiempo de abordar. En cualquier caso, queda eliminada la posibilidad de que sea la dimensión de género la que pueda darnos la clave para comprender esta concentración de universitarios en los grupos de administrativos y personal de servicios. 
Tabla 3. Proporción de mujeres ocupadas universitarias en sector alojamiento, en cada grupo de condición socioeconómica, 2009

\begin{tabular}{|l|r|}
\hline Empleadores & \multicolumn{2}{|c|}{40} \\
\hline Autónomos & \multicolumn{2}{|c|}{70,} \\
\hline Directivos & \multicolumn{2}{|c|}{68,} \\
\hline Profesionales & \multicolumn{2}{|c|}{} \\
\hline Administrativos & \multicolumn{2}{|c|}{15,} \\
\hline Resto personal servicios & \multicolumn{2}{|c|}{59,} \\
\hline Media en el colectivo & 5 \\
\hline & \multicolumn{2}{|c|}{63,} \\
\hline
\end{tabular}

Fuente: Elaboración propia con microdatos de EPA

\section{Reflexiones finales}

En primer lugar, el crecimiento del sistema educativo español ha facilitado la incorporación de activos universitarios al sector turístico, con una intensidad mayor que en el conjunto de la población ocupada. Y más dinámico incluso que el propio crecimiento del sector.

En segundo lugar, y pese a la continuidad de la estructura ocupacional en el sector a lo largo de veinte años, ha mejorado la proporción de profesionales en el mismo. Pero el destino de la mayoría de los titulados universitarios no son los puestos de empleadores, directivos y profesionales, sino los puestos administrativos y de servicios. En tercer lugar, esta situación no parece guardar relación con la habitual segregación laboral por géneros.

En cuarto lugar, cualquier reflexión sobre la productividad en el sector debería considerar no sólo la cantidad de personas con títulos de formación profesional o universitaria, sino el porcentaje de universitarios en los puestos directivos y profesionales. En gran medida, depende de ellos la reorganización estratégica eficiente de las empresas. Pese a la creciente importancia de la flexibilidad y de la polivalencia en las empresas, muchos puestos siguen observando contenidos y 
funciones que se explican por el mismo puesto y no únicamente por las características individuales de quienes los ocupan.

En quinto lugar, la comprensión de los procesos que afectan a los mercados de trabajo requiere de variables de carácter institucional, como el crecimiento de los activos egresados gracias al desarrollo del sistema educativo, y el discurso público que subraya la importancia de la educación en términos económicos; ello parece afectar a la segmentación tradicional de mercados de trabajo. El crecimiento de activos universitarios favorece en el sector turístico la incorporación de más profesionales, con lo que debilita parcialmente esta deficiencia tradicional en el sector. Aunque la mayor parte de ellos permanezca en los ámbitos centrales de la estructura ocupacional.

Sin embargo, queda sin resolver por qué se produce esta situación, la relativa al incremento de universitarios que favorece más a administrativos y personal de servicios que a los directivos, profesionales y empleadores. Aquí finalizamos avanzando tres hipótesis:

1. Los mecanismos de contratación en las empresas turísticas no priorizan necesariamente los títulos universitarios para directivos y profesionales. Conviviendo dichos títulos con otros criterios como experiencia o cercanía a la propiedad de las empresas. El análisis del capital humano (entendido como número de años de estudio) y la baja rentabilidad que parece encontrar en el sector turístico tendrían que ver con la conjunción de características estructurales en el mismo, que lleva a preferencias de contratación donde los títulos compiten con otros componentes o características de los individuos. Esta hipótesis es coherente con diversas investigaciones de carácter cualitativo (Brotons Martínez, 2012; Marrero Rodríguez, 2004). Y se resumiría en la idea de que en el sector turístico el título universitario convive con otras señales para las prácticas de contratación y promoción. Estas señales serían la experiencia, la pertenencia a redes sociales o determinados activos que no requieren necesariamente del sistema educativo (idiomas, por ejemplo).

2. Otra opción es considerar que las empresas han aprovechado el incremento de activos universitarios para incorporarlos en diversos niveles, incluso en aquellos casos en que no se necesita de tales títulos. En este caso estaríamos en el terreno de la sobreeducación (Blázquez Cuesta y Mora, 2010). Aunque esta opción no explica la baja proporción de universitarios en los grupos de directivos y profesionales del sector estudiado.

3. O quizá las empresas están aprovechando este incremento de egresados universitarios para incorporarlos a espacios del organigrama empresarial, al tiempo que proceden a cambios organizativos internos que no se reflejan en las denominaciones de los puestos de los individuos. En este caso, se produciría un desfase entre títulos, competencias desarrolladas y puesto reconocido -lo que además complicaría analizar la rentabilidad de la educación formal-. 
En cualquier caso, estas tres hipótesis requieren una aproximación metodológica complementaria y diferente a la que proporciona la Encuesta de Población Activa. Que pueda adentrarse más en las dinámicas internas de las empresas y en la toma de decisiones de selección y movilidad del personal.

Los resultados de este trabajo pueden contextualizar mejor los resultados de otras publicaciones como las de Cervera-Taulet y Ruiz-Molina (2008), donde se concluye que pese a la relativamente alta inserción laboral de los egresados en los títulos de turismo de la Universidad de Valencia, éstos consideran que muchos de los contenidos de las titulaciones cursadas son inútiles en su trabajo. Todo ello tendría que ver con la indefinición de muchos de los empleos turísticos al tiempo que se requieren cada vez más de titulados universitarios en las empresas turísticas. Así como en el trabajo de Marchante Mera y otros (2004), que aunque concluyen que la sobreeducación es menor en el sector que lo que se evidencia en otros estudios sobre mercado de trabajo en España; sin embargo, los niveles de sobreducación más elevados aparecen entre los universitarios. También resulta coherente con su idea de que las empresas se apoyan más en la experiencia que en los créditos formativos de los individuos. Por otro lado, como se planteó al comienzo de este texto, también complementa el planteamiento de trabajos como los de Lillo-Bañuls y Casado Díaz (2011). Pues considerando los puestos, más que a los individuos es posible comprender un poco mejor la situación de los bajos salarios de los universitarios en el sector turístico. Una mayor profundización en estas situaciones laborales podrá aclarar las complejas dinámicas que se han observado en este texto.

\section{Bibliografía}

Airey, D.; Frontistis, A. (1997). Attitudes to careers in tourism: An anglo greek comparison. Tourism Management. Vol. 18 Núm. 3, 149-158.

Álvarez Aledo, C. (1996). El impacto de la contratación temporal sobre el sistema productivo español. Madrid: Consejo Económico y Social.

Baum, T. (1995). Managing human resources in the European tourism and hospitality industry: A strategic approach. London-New York: Chapman \& Hall.

Baum, T. (2007). Human resources in tourism: Still waiting for change. Tourism Management. Vol. 28 Núm. 6, 1383-1399.

Blázquez Cuesta, M.; Mora, T. (2010). Overeducation and job mobility: Evidence from young recent graduates in Catalonia. Revista de Economía Laboral. Vol. 7 Núm. 1, 63-83. 
Brotons Martínez, M. (2012). Análisis comparativo de la formación reglada en turismo: Benidorm y el capital humano hotelero. Investigaciones Turísticas. Núm. 3, 120-132.

Ceballos Hernández, C.; Arias Martín, C.; Ruiz Jiménez, A.; Sanz Domínguez, C.; Vázquez Bermúdez, I. (2010). La formación en turismo en España: Pasado, presente y futuro en el nuevo espacio europeo de educación superior. Cuadernos de Turismo. Núm. 5, 45-68.

Cervera-Taulet, A.; Ruiz-Molina, M.E. (2008). Tourism education: a strategic analysis model. Journal of Hospitality, Leisure, Sport and Tourism Education. Vol. 7 Núm. 2, 59 - 70.

Eurofound (2009). Comprehensive sectoral analysis of emerging competencies and economic activities in the European Union.

García Manjón, J.V.; Pérez López, M.D.C. (2008). El grado en turismo: Un análisis de las competencias profesionales. Cuadernos de Turismo. Núm. 21, 67-84.

García Pozo, A.; Campos Soria, J. A.; Sánchez Ollero, J. L.; Marchante Lara, M. (2011). Capital humano y salarios en la hostelería española: Un análisis regional. Revista de Estudios Regionales. Núm. 91, 71-96.

Ladkin, A. (2011). Exploring tourism labor. Annals of Tourism Research. Vol. 38 Núm. 3, 1135-1155.

Ladkin, A.; Riley, M. (1996). Mobility and structure in the career paths of UK hotel managers: a labour market hybrid of the bureaucratic model?. Tourism Management. Vol. 17 Núm. 6, 443-452.

Lam, T. (2002). New employees' turnover intentions and organizational commintment in the Hong Kong hotel industry. Journal of Hospitality and Tourism Research. Vol. 26 Núm. 3, 217-234.

Lee, C.; Kang, S. (1998). Measuring earnings inequality and median earnings in the tourism industry. Tourism Management. Vol. 19 Núm. 4, 341-348.

Lennon, J. J.; Wood, R.C. (1989). The sociological analysis of hospitality labour and the neglect of accommodation workers. International Journal of Hospitality Management. Vol. 8 Núm. 3, 227-235.

Lever, A. (1987). Spanish tourism migrants: The case of Lloret de Mar. Annals of Tourism Research. Vol. 14 Núm. 4, 449-470. 
Lillo Bañuls, A.; Casado Díaz, J.M. (2011). Capital humano y turismo: rendimiento educativo, desajuste y satisfacción laboral. Estudios de economía aplicada. Vol. 29 Núm. 3, 755-780.

Liu, A.; Wall, G. (2005). Human resources development in China. Annals of Tourism Research. Vol. 32 Núm. 3, 689-710.

Lucas, R. (2004). Employment relations in the hospitality and tourism industries. London-New York: Routledge.

Mallorquí Ruscalleda, N. (2007). La mayor dotación de capital humano y sus problemas de adaptación en el mercado de trabajo español, 1986-2005. ¿Por qué continúan existiendo tantas diferencias con Europa?. Working Papers (Universitat Autònoma de Barcelona. Unitat d'Història Econòmica), Núm. 3.

Marchante Mera, A.J. y otros (2004). Desajuste educativo y movilidad laboral de los trabajadores de hostelería en Andalucía. Revista de Estudios Regionales. Núm. 069, 57-88.

Marrero Rodríguez, J.R. (2004). La estructura y dinámica de los mercados de trabajo en las actividades de servicios: el caso del sector turístico canario. Las Palmas de Gran Canaria: Servicio de Publicaciones del Cabildo Insular de Gran Canaria.

Mazón Martínez, T. y otros (2009). Turismo, urbanización y estilos de vida: las nuevas formas de movilidad residencial. Barcelona: Icaria.

Pizam, A. (1999). The state of travel and tourism human resources in Latin America. Tourism Management. Vol. 20 Núm. 5, 575-586.

Pries, L. (1988). Calificación, relaciones laborales y mercado de trabajo: El concepto de "estrechez del ámbito empresarial" en España. Revista Española de Investigaciones Sociológicas. Núm. 41, 81-114.

Riley, M.; Ladkin, A.; Szivas, E. (2002). Tourism employment. Analysis and planning. England: Channel View Publications.

Riley, M.; Szivas, E. (2003). Pay determination. A socioeconomic framework. Annals of Tourism Research. Vol. 30 Núm. 2, 446-464.

Rodríguez Rodríguez, J.; Arroyo Varela, S. R. (2000). La gestión de los recursos humanos a través de la flexibilidad laboral interna. Estudio empírico de los hoteles de la Costa del Sol. Papers de Turisme. Núm. 28, 67-95. 
Sala, G. (2011). Approaches to skills mismatch in the labour market: a literature review. Papers. Vol. 96 Núm. 4, 1025-1045.

Sarrió, M.; Baberá, E.; Ramos, A.; Candela, C. (2002). El techo de cristal en la promoción profesional de las mujeres. Revista de Psicología Social. Vol. 17 Núm 2, 167-182.

Servicio Público de Empleo Estatal (2011). Características socio-demográficas y perfiles competenciales de los trabajadores del sector turismo. Madrid: Ministerio de Trabajo e Inmigración.

Serrano Martínez, J.M. (2011). La vivienda en España dentro de un nuevo modelo económico. El papel y los límites del sector turístico ¿hacia el declinar de un ciclo?. Cuadernos de Turismo. Núm. 27, 855-874.

Sheldon, P. J. (1989). Professionalism in tourism and hospitality. Annals of Tourism Research. Vol. 16 Núm. 4, 492-503.

Van den Berghe, P.L. (1992). Tourism and the ethnic division of labor. Annals of Tourism Research. Vol. 19 Núm. 2, 234-249.

Villa, P. (1990). La estructuración de los mercados de trabajo. La siderurgia y la construcción en Italia. Madrid: Ministerio de Trabajo y Seguridad Social.

Wirth, L. (2002). Romper el techo de cristal. Las mujeres en puestos de dirección. Madrid: Ministerio de Trabajo y Asuntos Sociales.

Witz, A. (1992). Professions and patriarchy. London-N.York: Routledge. 